Nota científica

(Short communication)

\title{
REGISTROS DE MAMÍFEROS (MAMMALIA: DIDELPHIMORPHIA, ARTIODACTYLA, CARNIVORA, CINGULATA, LAGOMORPHA, PILOSA Y RODENTIA) EN RESERVAS NATURALES PRIVADAS DE GUATEMALA
}

\author{
RECORD OF MAMMALS (MAMMALIA: DIDELPHIMORPHIA, ARTIODACTYLA, \\ CARNIVORA, CINGULATA, LAGOMORPHA, PILOSA AND RODENTIA) IN PRIVATE \\ NATURAL RESERVES IN GUATEMALA
}

\section{BÁRBARA ISABELA ESCOBAR-ANLEU, ,,2,3,* CÉSAR ESTUARdo FUENTES-MONTEJO ${ }^{3}$ y DANIEL ARIANO-SÁNCHEZ ${ }^{4,5}$}

\footnotetext{
${ }^{1}$ Programa de Monitoreo de Biodiversidad, Asociación de Reservas Naturales Privadas de Guatemala, 5ta Calle 0-50 zona 14, Edificio Anacafé, Ciudad de Guatemala.

${ }^{2}$ Instituto Internacional en Conservación y Manejo de Vida Silvestre, Universidad Nacional, Heredia Costa Rica.

${ }^{3}$ Escuela de Biología, Universidad de San Carlos de Guatemala, Edificio T-10, Ciudad Universitaria zona 12, C. P. 01012 Ciudad de Guatemala, Guatemala.

${ }^{4}$ Departamento de Biología, Universidad del Valle de Guatemala, 11 Calle 15-79 zona 15 V.H. III, Ciudad de Guatemala.

${ }^{5}$ Reserva Natural para la Conservación del Heloderma y el Bosque Seco, Asociación Zootropic, El Arenal, Cabañas, Zacapa, Guatemala.

* Autora de correspondencia: <bisa_e8a9@hotmail.com>.
}

Recibido: 20/04/2016; aceptado: 24/05/2017

Editor Responsable: Alberto González Romero.

Escobar-Anleu, B. I., Fuentes-Montejo, C. E. y Ariano-Sánchez, D. (2017). Registros de mamíferos (Mammalia: Didelphimorphia, Artiodactyla, Carnivora, Cingulata, Lagomorpha, Pilosa y Rodentia) en reservas naturales privadas de Guatemala. Acta Zoológica Mexicana (n.s.), 33(2), 389-393.

RESUMEN. Se documentan 25 especies de mamíferos en las reservas naturales privadas de Guatemala, principalmente usando cámara trampa, desde mayo 2010 a octubre 2015. Las especies pertenecen a los órdenes Carnivora (13), Rodentia (3), Didelphimorphia (3), Artiodactyla (2), Lagomorpha (2), Cingulata (1) y Pilosa (1). De las especies registradas, 21 se encontraron en localidades no documentadas en la literatura o en base de datos disponibles en línea, lo que refleja la importancia de hacer investigaciones en las reservas naturales privadas.

Los mamíferos son organismos muy amenazados, siendo algunas de las principales amenazas la pérdida de hábitat, la sobreexplotación y los cambios en el uso del suelo (Ahumada et al., 2011). Una de las estrategias más utilizada para minimizar la pérdida de biodiversidad es el establecimiento de sistemas de áreas protegidas (Ur-
Escobar-Anleu, B. I., Fuentes-Montejo, C. E., \& Ariano-Sánchez, D. (2017). Record of mammals (Mammalia: Didelphimorphia, Artiodactyla, Carnivora, Cingulata, Lagomorpha, Pilosa and Rodentia) in private natural reserves in Guatemala. Acta Zoológica Mexicana (n.s.), 33(2), 389-393.

ABSTRACT. We documented 25 species of mammals in private natural reserves in Guatemala, mainly through camera traps, from May 2010 to October 2015. Species belong to the orders Carnivora (13), Rodentia (3), Didelphimorphia (3), Artiodactyla (2), Lagomorpha (2), Cingulata (1) and Pilosa (1). Of the species reported, 21 were found in locations not documented in the literature or on databases available online, which reflects the importance of doing research in private natural reserves.

quiza-Haas \& Koleff, 2011). En Guatemala, las reservas naturales privadas (RNP) están reconocidas como una categoría dentro del Sistema Guatemalteco de Áreas Protegidas (SIGAP) las cuales son destinadas voluntariamente por sus propietarios a la conservación (Consejo Nacional de Áreas Protegidas -CONAP-2007) (Fig. 1). A pesar de 


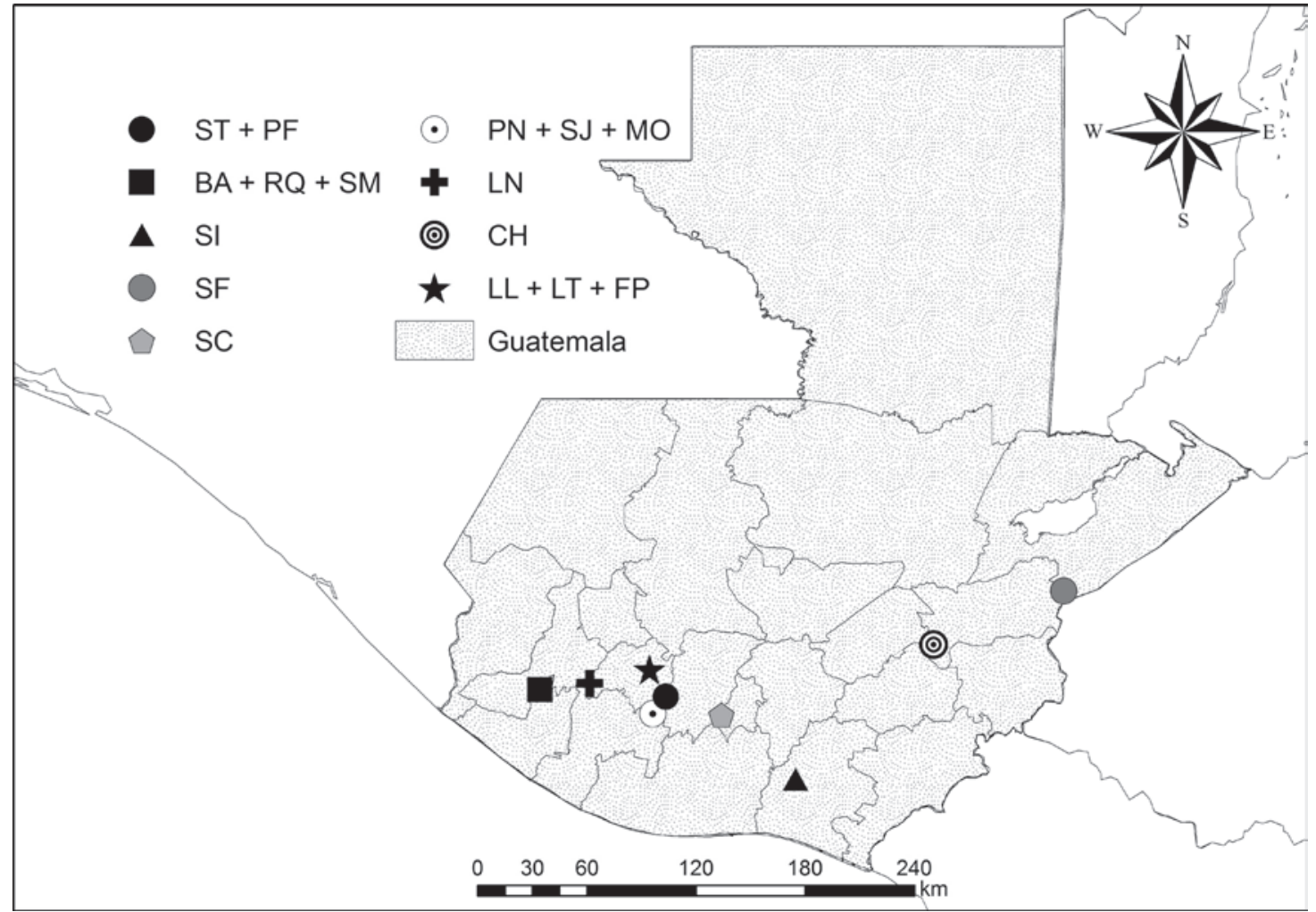

Figura 1. Mapa de Guatemala con distribución de las Reservas Naturales Privadas con registros de mamíferos. ST $+\mathrm{PF}=\mathrm{Santo}$ Tomás Pachuj y Pampojilá-Peña Flor; BA + RQ + SM= Buenos Aires, Rosario Quezada y Santa Margarita; SI= Santa Isabel; SF= San Francisco El Chorro; SC= Santa Catalina, Anexo La Joya Carbonera y la Travesía, PN + SJ + MO= Panamá, San Jerónimo Miramar-Quixayá y Monte De Oro; LN= Las Nubes; CH= Reserva para la Conservación del Heloderma; LL + LT + FP= Laguna Lodge, Los Tarrales y Finca Patrocinio.

que su objetivo de conservación es claro, los esfuerzos de investigación de la diversidad biológica presente en estas reservas son reducidos y están desactualizados. Existen listados generales de mamíferos de Guatemala, pero sin identificar su distribución en el SIGAP (McCarthy \& Pérez, 2006; Kraker-Castañeda et al., 2016).

Para este estudio utilizamos cámaras marca Bushnell y en todos los casos fueron operadas por, al menos, uno de nosotros. Estas permanecieron activas ininterrumpidamente durante las fechas indicadas (Cuadro 1). Debido a que los datos provienen de distintos proyectos, la obtención de los registros ha sido mediante diferentes diseños, todos con el objetivo de caracterizar las especies de mamíferos presentes en cada reserva. Aunque el esfuerzo de captura fue distinto según el proyecto del cual lo obtuvimos, lo estimamos en 2256 días-cámara trampa (Fig. 2). Además, realizamos revisión de literatura (Ordóñez-Garza et al., 2008; McCarthy \& Pérez, 2006; Kraker-Castañeda et al., 2016), colecciones de referencia nacionales (Museo de Historia Natural de la Universi- dad de San Carlos de Guatemala -MUSHNAT-USACy Colecciones Biológicas de la Universidad del Valle de Guatemala -CB-UVG) y bases de datos internacionales (Global Biodiversity Information Facility - GBIF [2015]) y el Smithsonian National Museum of Natural History -NMNH- [2016]) para determinar localidades no reportadas previamente para las especies. Se revisaron los listados oficiales de la Convención sobre el Comercio Internacional de Especies Amenazadas de Fauna y Flora Silvestres (CITES), el CONAP y la Unión Internacional para la Conservación de la Naturaleza (UICN) para determinar si las especies encontradas están bajo algún grado de amenaza. Adicionalmente, registramos algunos ejemplares encontrados muertos en las RNP estudiadas tales como Didelphis virginiana (2015 en El Chalí, Panajachel, Sololá), Puma concolor (2010 en San Jerónimo Miramar-Quixayá, Patulul, Suchitepéquez), Canis latrans (2016 en Reserva Heloderma, Cabañas, Zacapa) y Mustela frenata (2016 en Reserva Heloderma, Cabañas, Zacapa). Los primeros dos ejemplares fueron deposita- 
Cuadro 1. Reservas Naturales Privadas de Guatemala estudiadas por fototrampeo para el registro de mamíferos.

\begin{tabular}{|c|c|}
\hline Localidad & Período de fototrampeo \\
\hline -Rosario Quezada, Flores Costa Cuca, Quetzaltenango, 14.666, -91.799997, 537 msnm & $13 / 10-05 / 11 / 2013$ \\
\hline -San Francisco El Chorro, Los Amates, Izabal, 15.127639, -89.146306, 688 msnm & $22 / 07-07 / 08 / 2014$ \\
\hline $\begin{array}{l}\text {-Santa Catalina, Anexo La Joya Carbonera y la Travesía San Miguel Dueñas, Sacatepéquez, } \\
\text { 14.514472, -90.838056, } 1806 \text { msnm }\end{array}$ & $16-31 / 10 / 2014$ \\
\hline -Santa Isabel, Pueblo Nuevo Viñas, Santa Rosa, 14.196444, -90.472583, 1393 msnm & $31 / 07-14 / 08 / 2014$ \\
\hline -Monte de Oro, Santiago Atitlán, Sololá, 14.552167, -91.242389, 963 msnm & $16-30 / 12 / 2014$ \\
\hline -Santo Tomás Pachuj, San Lucas Tolimán, Sololá, 14.604222, -91.114028, 1338 msnm & $27 / 03-11 / 04 / 2014$ \\
\hline -Los Tarrales, Patulul, Suchitepéquez, 14.740083, -91.194333, 1698 msnm & $01-15 / 12 / 2014$ \\
\hline -Finca Panamá, Santa Bárbara, Suchitepéquez, 14.520222, -91.178194, 922 msnm & $06-20 / 02 / 2014$ \\
\hline -Las Nubes, San Francisco Zapotitlán, Suchitepéquez, 14.670306, -91.490556, 1470 msnm & $03-17 / 07 / 2014$ \\
\hline -San Jerónimo Miramar-Quixayá, Patulul, Suchitepéquez, 14.539806, -91.138194, 901 msnm & $15-30 / 04 / 2014$ \\
\hline -Reserva para la Conservación del Heloderma, Cabañas, Zacapa, 14.863722, -89.789111, 644 msnm & $\begin{array}{l}\text { 15/06/2013 - 15/06/2015 } \\
\text { (20 días cada mes) }\end{array}$ \\
\hline
\end{tabular}

Cuadro 2. Especies de mamíferos documentadas en 16 Reservas Naturales Privadas de Guatemala

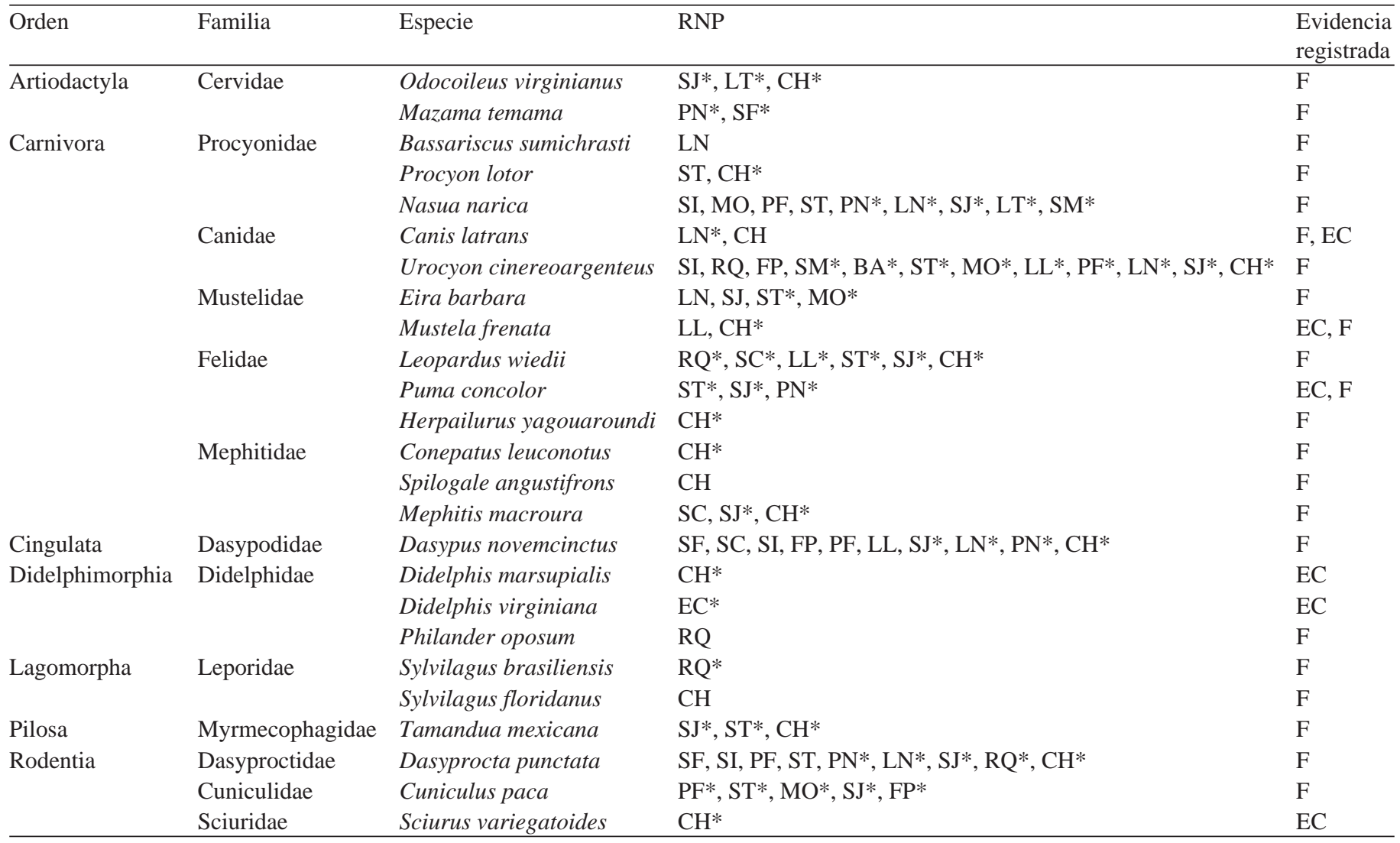

RNP = reserva natural privada, F = Fotográfico, EC = Ejemplar colectado, BA = Buenos Aires, CH = Reserva para la Conservación del Heloderma, EC = El Chalí, FP = Finca Patrocinio, LN = Las Nubes, LL = Laguna Lodge, $\mathrm{LT}=$ Los Tarrales, $\mathrm{MO}=$ Monte De Oro, $\mathrm{PF}=$ Pampojilá-Peña Flor, $\mathrm{PN}=$ Panamá, $\mathrm{RQ}=$ Rosario Quezada, SC = Santa Catalina, Anexo La Joya Carbonera y la Travesía, SF = San Francisco El Chorro, SI = Santa Isabel, SJ = San Jerónimo Miramar-Quixayá, SM = Santa Margarita, ST = Santo Tomás Pachuj, * = reservas naturales privadas localizadas en departamentos sin registros previos de la especie. 

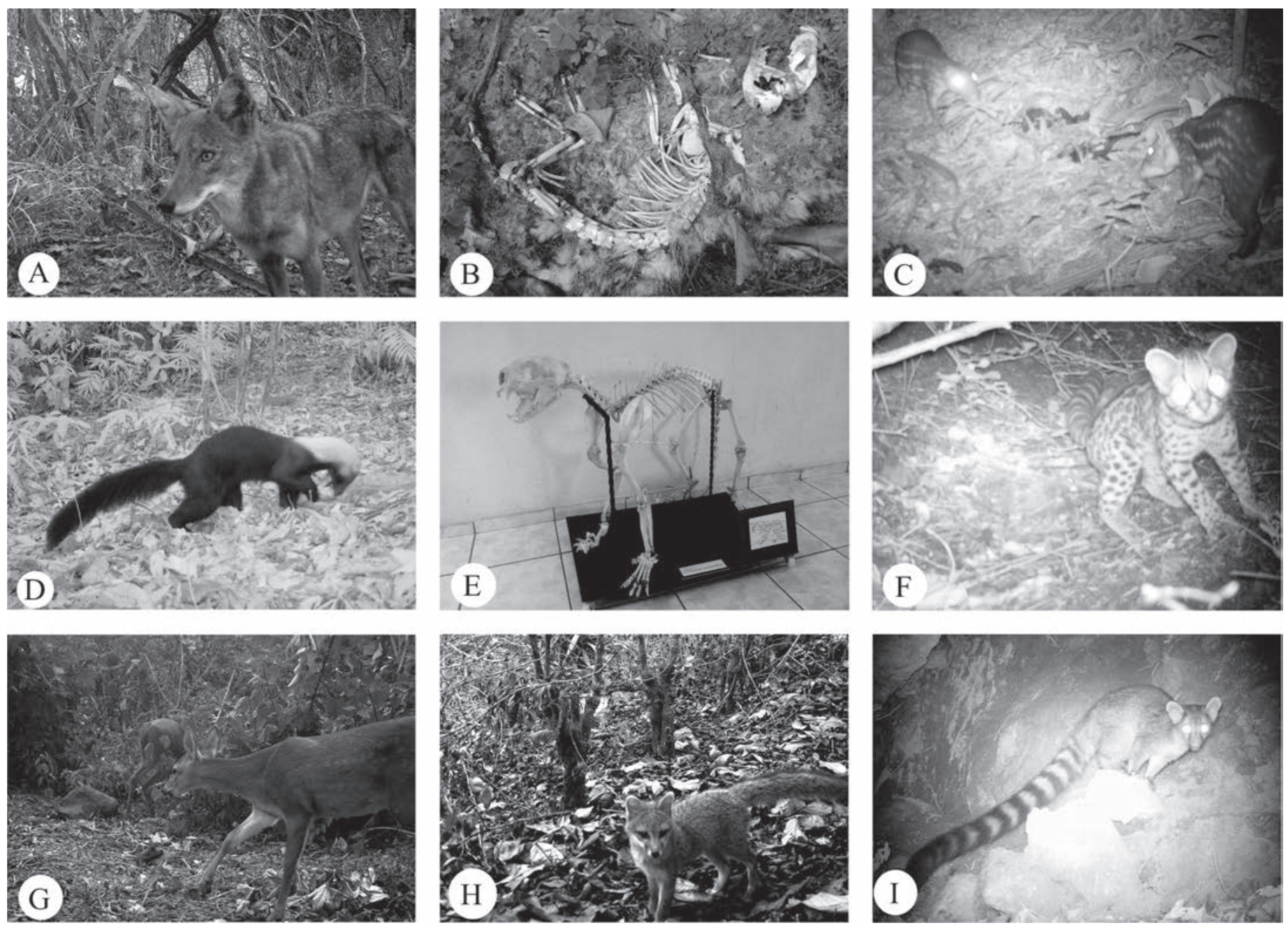

Figura 2. Especies de mamíferos registrados en Reservas Naturales Privadas. A) Canis latrans (ZOOTROPIC / G. Salazar), B) Didelphis virginiana (B. Escobar-Anleu), C) Cuniculus paca (ARNPG / B. Escobar-Anleu), D) Eira barbara (ARNPG / B. Escobar-Anleu), E) Puma concolor (J. Calderón Solís), F) Leopardus wiedii (ARNPG / B. Escobar-Anleu), G) Odocoileus virginianus (ARNPG / B. Escobar-Anleu), H) Urocyon cinereoargenteus (ARNPG / B. Escobar-Anleu), I) Bassariscus sumichrasti (ARNPG / B. Escobar-Anleu).

dos en el MUSHNAT- USAC y los dos segundos en la CB-UVG.

En el Cuadro 2, presentamos las especies de mamíferos que documentamos en distintas reservas naturales privadas. La mayoría de especies encontradas están incluidas en la categoría de "Preocupación menor" de la lista roja de la UICN, a excepción de L wiedii (Casi Amenazada) y $M$. temama (Información insuficiente). Además, tres de las especies registradas (L. wiedii, $P$. concolor y $H$. yaguaroundi) se encuentran en el Apéndice I de CITES. A nivel nacional, 14 especies están en la categoría 3 de la Lista de Especies Amenazadas de CONAP (B. sumichrasti, $P$. lotor, $N$ narica, C. latrans, D. punctata, D. marsupialis, D. virginiana, C. paca, O. virginianus, E. barbara, S. va- riegatoides, S. brasiliensis, S. floridanus y T. mexicana) mientras que L. wiedii, $P$. concolor, $H$. yagouaroundi y M. temama están en la categoría 2.

Las reservas naturales privadas tienen un gran potencial para reforzar y complementar los sistemas gubernamentales de áreas protegidas, conservando ecosistemas que se encuentran ausentes o poco representados en el sistema público (Sepúlveda, 2002). Además, aportes como el nuestro permiten evidenciar la importancia de promover la conservación e investigación en tierras privadas. Estas áreas consisten generalmente de sistemas agroforestales o remanentes pequeños de bosque natural los cuales proveen una alternativa útil como herramienta de conservación en paisajes fragmentados. Las tierras priva- 
das manejadas con criterios de sostenibilidad contribuyen a la conservación de la mastofauna guatemalteca.

AGRADECIMIENTOS. A la ARNPG y a Zootropic por el apoyo logístico y de equipo, así como a los propietarios y trabajadores de las distintas reservas. A Sara Ortiz, Gilberto Salazar y Erik López por su colaboración en parte del trabajo de campo. A José Mora por sus comentarios sobre el documento. Al personal de MUSHNAT, así como de las Colecciones Biológicas de la UVG.

\section{LITERATURA CITADA}

Ahumada, J. A., Silva, C. E., Gajapersad, K., Hallam, C., Hurtado, J., Martin, E., McWilliam, A., Mugerwa, B. O’Brien, T., Rovero, F. Sheil, D. Spironello, W. R., Winarni, N. \& Andelman, S. J. (2011). Community structure and diversity of tropical forest mammals: data from a global camera trap network. Philosophical Transactions of the Royal Society, 366, 2703-2711.

Consejo Nacional de Áreas Protegidas -CONAP (2007). Guía para establecer una reserva natural privada. Guatemala: CONAP.
GBIF Secretariat. (2015). GBIF: Global Biodiversity Information Facility, datasets from Guatemala, Available at: http://www.gbif. org/country/GT/summary

Kraker-Castañeda, C., Pérez, S. G., Cajas-Castillo, J. O. \& Echeverría-Tello, J.L. (2016). Lista actualizada de los murciélagos (Mammalia, Chiroptera) de Guatemala. Revista Mexicana de Biodiversidad, 87, 409-416.

McCarthy, T. \& Pérez, S. (2006). Land and Freshwater Mammals of Guatemala: faunal documentation and diversity. Pp. 625-674. In: E. Cano (Ed.). Biodiversidad de Guatemala. Universidad del Valle de Guatemala, Guatemala.

Ordóñez-Garza, N., Bulmer, W., Eckerlin, R. P. \& Matson, J. O. (2008). Coyotes (Canis latrans) in Guatemala. The Southwestern Naturalist, 53, 507-509.

Sepúlveda, C. (2002). Áreas protegidas y territorio: la conectividad que falta. Ambiente y Desarrollo XVII, 118-124

Urquiza-Haas, T., \& Koleff, P. (2011). Sitios prioritarios para la conservación de mamíferos terrestres: evaluación de los criterios de selección de indicadores. Pp. 131-150. In: P. Koleff, \& T. Urquiza-Haas (Eds.). Planeación para la conservación de la biodiversidad terrestre en México: retos en un país megadiverso. CONABIO-CONANP-SEMARNAT, México, D. F. 